\title{
Electroencephalography (EEG) Spectral Edge Frequency for Assessing the Sedative Effect of Acupuncture in Dogs
}

\author{
Min-Su KIM ${ }^{1)}$ and Tchi-Chou NAM ${ }^{1) *}$ \\ ${ }^{1)}$ Department of Veterinary Surgery, College of Veterinary Medicine, Seoul National University, San 56-1, Sillim 9-dong, Gwanak-gu, \\ Seoul, 151-742, Korea
}

(Received 31 August 2005/Accepted 21 December 2005)

\begin{abstract}
Electroencephalography (EEG) is an effective method for the evaluation of sedation or anesthesia. The purpose of this study was to examine the sedative effect of acupuncture by electroencephalographic spectral edge frequency (SEF) in Miniature Schnauzer dogs (4.2-6.1 kg, 1-2 years old). The acupuncture points "GV20 and Yintang” were applied for 20 min. Sedation level was assessed before, during, and after acupuncture by spectral edge frequency 95 values and the Ramsay sedation score. The spectral edge frequency 95 values were significantly reduced during acupuncture on GV20 or Yintang point and returned to the baseline values after acupuncture releasing. The Ramsay sedation score (RSS) also showed the acceptable sedation level during acupuncture. It was concluded that an acupuncture application at GV20 or Yintang point used in the present study would be a valuable method to induce the sedation in dogs. KEY WORDS: acupuncture, canine, spectral edge frequency.
\end{abstract}

Sedation is a state of mild central depression accompanied by drowsiness $[6,10]$. In veterinary field, sedation is required to produce behavior modification, stabilization, and muscle relaxation [13]. The primary method of sedation is the use of sedatives such as phenothiazine derivatives, benzodiazepines, or $\alpha_{2}$-adrenergic agonists. However, these drugs may be prolonged recovery time and are dangerous to treat for aged or diseased animals [11]. Recently it has been reported that nonpharmacological methods like an acupressure or acupuncture can induce sedation and provide relief from stress $[12,17,18]$. The assessment methods of sedation are verbal stress scale (VSS), Ramsay sedation score (RSS) and EEG [1, 7, 15]. The activity of mental depression or behavioral changes by acupuncture is related to the central nervous system (CNS) activity in brain [16]. The EEG may be a valuable tool in the evaluation of central nervous system activity $[8,12]$. The spectral edge frequency (SEF) is one of the important evaluation parameter of the EEG.

SEF 95 is determined by the $95 \%$ of the power spectral density and is used for assessing depth of sedation and anesthesia $[13,14]$. The present study was performed to investigate the sedative effect of acupuncture applied at the GV20 or Yintang point on the SEF95 and on the RSS in conscious dogs.

Twelve healthy intact male Miniature Schnauzer dogs (4.2-6.1 kg, 1-2 years old) without neurological abnormalities and disease were used for the study. This study adhered to the strict guidelines of the "Guide for the Care and Use of Laboratory Animals” of Seoul National University (Seoul, South Korea). Dogs were assigned randomly to four study groups ( $n=20 /$ group). Dogs were repeatedly used with a

\footnotetext{
* Correspondence to: Prof. Nam, T.-C., Department of Veterinary Surgery, College of Veterinary Medicine, Seoul National University, San 56-1, Sillim 9-dong, Gwanak-gu, Seoul, 151-742, Korea.
}

washout period of 2 weeks. The experimental groups were GV20 group, Yintang group, GV20 + Yintang group, and control group. The acupoint GV20 is located at the vertex on the midline, the Yintang point is midway between the medial ends of the two eyebrows. In traditional medicine, the GV20 point and Yintang point has been known to provide relief from stress and calms the spirit [5, 9]. The control group was received acupuncture in non-acupuncture point located $2 \mathrm{~cm}$ lateral from the lateral end of the right eyebrow [15]. All dogs were at rest for $1 \mathrm{hr}$ before experimentation. For the all groups, one stainless steel needle (32 gauge, $15 \mathrm{~mm}$ long, Haeng Lim Seo Won, Seoul, Korea) was inserted on the each point and was kept for $20 \mathrm{~min}$. To obtain the electroencephalogram activity, a computerized EEG (Model: QEEG-8, LXE3208, LAXTHA Inc., Korea) was used with 8 channel simultaneous recording and visualization in different monopolar montages from subdermal needle electrodes. In this study, EEG needles (LAXTHA Inc., Korea) were set subcutaneously at the middle, right and left frontal (FV, FR, and FL, respectively), right and left parietal (PR and PL, respectively), and middle, right and left occipital (OV, OR, and OL, respectively) areas. Ground and reference electrodes were set below the both ear. A method of standardized placement of EEG electrodes similar to the 10-20 international system used for the human being was used [2]. The low-frequency filter was set at 2 $\mathrm{Hz}$, and the high-frequency setting was $50 \mathrm{~Hz}$. After the electrodes were placed at appropriate sites, the first $10 \mathrm{~min}$ were spent allowing the dog to stay. A calm environment was obtained and recording was started. Measurements were made at time point a l (Fig. 1). Before (a) and after (l) acupuncture on the each point, EEG recording was determined the five times with intervals of $2 \mathrm{~min}$, the mean value was taken. During acupuncture period (b-k), EEG recording were measured at intervals $2 \mathrm{~min}$ for duration of $20 \mathrm{~min}$. At these time points, the experimental dogs were also assessed 


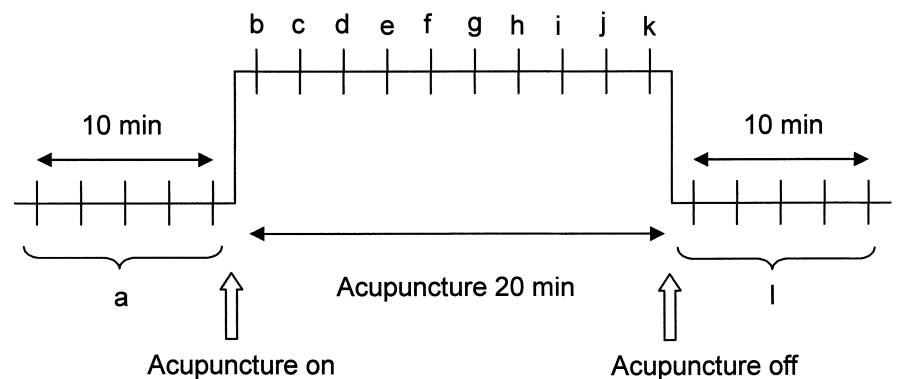

Fig. 1. Experimental procedure. Acupuncture procedure and measuring point before (a) during (b-k) and after (l) acupuncture. a, l : average of 5 times per every 2 min, b-k : 10 times per every 2 min.

Table 1. Time related spectral edge frequency $95(\mathrm{~Hz})$ changes in 4 groups

\begin{tabular}{cllcc}
\hline \multirow{5}{*}{ Time } & \multicolumn{4}{c}{ spectral edge frequency $95(\mathrm{~Hz}$ changes } \\
& \multicolumn{4}{c}{ Group } \\
& GV20 & \multicolumn{1}{c}{ Yintang } & GV20 \pm Yintang & Control \\
\hline a & $41.83 \pm 2.71$ & $41.21 \pm 3.18$ & $42.01 \pm 1.71^{\#, *}$ & $41.38 \pm 2.70$ \\
b & $40.21 \pm 4.91^{\#}$ & $39.66 \pm 6.12^{\#, *}$ & $40.83 \pm 1.68^{\#, *}$ & $42.97 \pm 2.63$ \\
c & $40.20 \pm 6.42^{\#, *}$ & $39.34 \pm 3.40^{\#, *}$ & $39.77 \pm 4.14^{\#, *}$ & $42.14 \pm 3.38$ \\
d & $38.84 \pm 6.30^{\#, *}$ & $39.26 \pm 5.39^{\#, *}$ & $38.34 \pm 2.66^{\#, *}$ & $43.38 \pm 3.05$ \\
e & $39.08 \pm 5.86^{\#, *}$ & $39.09 \pm 5.19^{\#, *}$ & $39.92 \pm 6.12^{\#, *}$ & $43.00 \pm 3.38$ \\
f & $38.47 \pm 5.91^{\#, *}$ & $39.16 \pm 3.97^{\#, *}$ & $38.46 \pm 3.49^{\#, *}$ & $42.52 \pm 2.32$ \\
g & $38.60 \pm 5.85^{\#, *}$ & $37.79 \pm 5.18^{\#, *}$ & $37.02 \pm 1.22^{\#, *}$ & $42.95 \pm 2.11$ \\
h & $37.89 \pm 6.07^{\#, *}$ & $39.13 \pm 3.82^{\#, *}$ & $37.80 \pm 3.90^{\#, *}$ & $42.84 \pm 2.14$ \\
i & $38.48 \pm 6.88^{\#, *}$ & $38.78 \pm 5.01^{\#, *}$ & $38.00 \pm 3.47^{\#, *}$ & $42.45 \pm 3.06$ \\
j & $38.39 \pm 4.93^{\#, *}$ & $38.84 \pm 4.68^{\#, *}$ & $39.49 \pm 1.80^{\#, *}$ & $43.07 \pm 2.51$ \\
k & $38.87 \pm 5.82^{\#, *}$ & $38.87 \pm 4.37^{\#, *}$ & $39.00 \pm 4.15^{\#, *}$ & $42.03 \pm 2.39$ \\
l & $42.32 \pm 1.94$ & $41.73 \pm 2.48$ & $42.44 \pm 1.19$ & $43.17 \pm 1.80$ \\
\hline
\end{tabular}

Spectral edge frequency 95 values (mean \pm SD) before (a), during (b-k) and after (l) acupuncture (cf. Fig. 1) recorded from the OV electrode in four groups (GV20, Yintang, GV20 + Yintang, and control) was observed. \# Significantly different from control, * Significant different from a $(p<0.05)$.

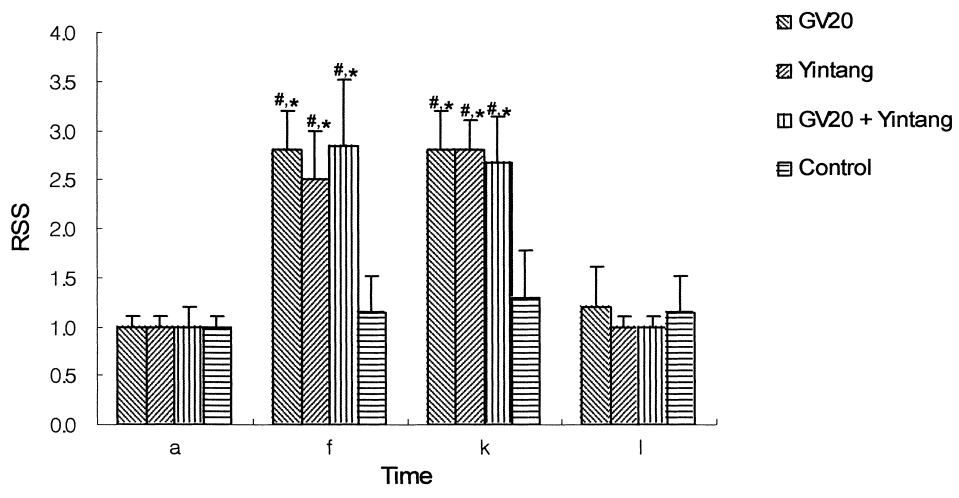

Fig. 2. Ramsay sedation score (RSS) values (mean SD) before (a), 10 min (f), 20 $\min (\mathrm{k})$ and after (l) acupuncture (cf. Fig. 1) in four groups were observed. \# Significantly different from control, * Significant different from a $(\mathrm{p}<0.05)$. 
the sedation level, based on the RSS from 1 (agitated) to 6 (unresponsive) [1].

Wilcoson's signed rank test was used to compare the baseline value in each group. The values between 4 groups were evaluated by Kruskal-Wallis test. The levels of significance were chosen as $p<0.05$. The following EEG data were shown at OV electrode.

The baseline SEF 95 values recorded for GV20 group, Yintang group, GV20 + Yintang group, and control group were similar. Before insertion of needle, the mean SEF 95 values ( \pm standard deviation (SD)) were $41.83 \pm 2.71$ (GV20 group), $41.21 \pm 3.18$ (Yintang group), $42.01 \pm 1.72$ (GV20 + Yintang group), and $41.38 \pm 2.70$ (control group), respectively. However, the SEF 95 values significantly decreased during acupuncture applied to the acupoint GV20 and Yintang in dogs $(p<0.05$, Table 1$)$. After 10 min acupuncture at the GV20 and Yintang point, the mean SEF 95 values were $38.47 \pm 5.91$ (GV20 group), $39.16 \pm 3.97$ (Yintang group), and $38.46 \pm 3.49$ (GV20 + Yintang group), respectively. The intragroup (GV20, Yintang, and GV20 + Yintang) analysis of SEF 95 values did not show any significant difference. After releasing the acupuncture at the acupoint GV20 and Yintang, SEF 95 was returned to the baseline values before stimulation. The SEF 95 values of control group were insignificantly changed during all the experimental period. RSS values were similar in the four groups before acupuncture and after leasing acupuncture. However, the RSS values were significantly increased during acupuncture at the GV20 and Yintang when compared to the RSS values before acupuncture ( $p<0.05$, Fig. 2$)$.

Based on the results, we know that acupuncture at the GV20 and Yintang induces sedation in dogs. Although the RSS assessment is the gold standard to assess the sedation, the method is a little subjective [1]. However, EEG is the one of the effective and objective methods to evaluate the sedation. EEG-derived parameters have been done for confirming the effect of anxiety, pain, and sedation induced by acupuncture [7, 12, 15]. Among the EEG parameters, the SEF 95 is mainly used to monitor the hypnotic effect of sedatives or anesthetics [12]. Our results demonstrated that acupuncture applied at the acupoint GV20 and Yintang decreased SEF 95 value. On the contrary, acupuncture applied at the non-acupoint did not influence on SEF 95 values. The control point was not related with any acupuncture point [15]. Many clinicians have usually used sedatives for relieving anxiety stress or sedation to animals. However, sedatives and opioids are associated with some complications such as respiratory depression, nausea, and vomiting [7]. Lately, non-pharmacological methods have been researched to prevent or treat pain and emesis [7]. Acupuncture treatment has been studied as a valuable treatment in improving the quality sleep, mental disorder and dysmenorrhea $[5,12]$. According to the view of traditional medicine, stimulation of GV20 and Yintang point may be used to release the anxiety or stress, in addition to treat the pain or mental convulsiveness [3]. In human, stimulation of the GV20 and Yintang point have been used to study the effect of sedation and the state of stress in conscious condition [4, $5,7,12]$. We also found that a drowsiness and sedation was induced by acupuncture applied at the acupoint GV20 and Yintang in this study.

In conclusion, the application of acupuncture on the GV20 and Yintang point showed a significant decrease in SEF 95 values and an acceptable sedation level in RSS value. In clinics, acupuncture applied at the GV20 and Yintang point would be useful to sedate in dogs.

\section{REFERENCES}

1. Bell, J. K., Laasch, H. U., Wilbraham, L., England, R. E., Morris, J. A. and Martin, D. F. 2004. Clin. Radiol. 59: 1106-1113.

2. Bergamasco, L., Accatino, A., Priano, L., Neiger-Aeschbacher, G., Cizinauskas, S. and Jaggy, A. 2003. Vet. J. 166: 58-66.

3. Chang, Y. H., Hsieh, M.T. and Cheng, J. T. 1996. Neurosci. Lett. 211: 121-124.

4. Chongcheng, X., Huansen, X., Qingchi, R., Yuande, C. and Dingli, L. 1985. Convuls. Ther. 1: 242-251.

5. Dos Santos, J. G. Jr., Tabosa, A., do Monte, F. H., Blanco, M.M., de Oliveira Freire, A. and Mello, L. E. 2005. Neurosci. Lett. 26: 234-238.

6. Dressler, O., Schneider, G., Stockmanns, G. and Kochs, E. F. 2004. Br. J. Anaesth. 93: 806-809.

7. Fassoulaki, A., Paraskeva, A., Patris, K., Pourgiezi, T. and Kostopanagiotou, G. 2003. Anesth. Analg. 96: 885-890.

8. Fox, M. W. 1963. Am. J. Vet. Res. 24: 1240-1247.

9. Han, C., Li, X., Luo, H., Zhao, X. and Li, X. 2004. J. Tradit. Chin. Med. 24: 172-176.

10. Herin, R. A., Purinton, P. T. and Fletcher, T. F. 1968. Am. J. Vet. Res. 29: 329-336.

11. Klemm, W. R. 1968. Am. J. Vet. Res. 29: 1267-1277.

12. Klemm, W. R. 1968. Am. J. Vet. Res. 29: 337-351.

13. Klemm, W. R. and Mallo, G. L. 1966. J. Am. Vet. Med. Assoc. 148: 1038-1042.

14. Martin-Cancho, M. F., Lima, J. R., Luis, L., Crisostomo, V., Ezquerra, L. J., Carrasco, M. S. and Uson-Gargallo, J. 2003. Am. J. Vet. Res. 64: 866-873.

15. Paraskeva, A., Melemeni, A., Petropoulos, G., Siafaka, I. and Fassoulaki, A. 2004. Am. J. Chin. Med. 32: 789-794.

16. Redding, R. W., Prynn, R. B. and Wagner, J. L. 1966. J. Am. Vet. Med. Assoc. 15: 141-149.

17. Tourai, K., Senba, H., Sasaki, N., Tokuriki, M., Ohashi, F., Takeuchi, A. and Usui, K. 1985. Nippon. Juigaku. Zasshi. 47: 459-463.

18. Yi, P. L., Tsai, C. H., Lin, J. G., Liu, H. J. and Chang, F. C. 2004. J. Biomed. Sci. 5: 579-590. 